\title{
Estimation of salt tolerance in Andrographis paniculata accessions using multiple regression model
}

\begin{abstract}
The complexity and polygenic nature of the salt tolerance trait in plants needs to develop a multiple indicator in the screening process. The mentioned issue led us to carry out an experiment to identify tolerant genotypes through multiple parameters in Andrographis paniculata. For this purpose, the 40-days seedlings were grown in different salinity levels (control, 4, 8, 12 and $16 \mathrm{dS}$ mī 1) on Hoaglandôs medium. The results indicated that salinity had a significant effect on the morphological, physiological and biochemical traits. All measured morphological traits, and chlorophyll, $\mathrm{K}+$ and $\mathrm{Ca} 2+$ content were significantly decreased with increasing salinity levels, while proline and $\mathrm{Na}+$ content increased. The present exploration revealed that, salt tolerance index (STI), using the multiple regression model, demonstrated a more stable trend than the single variable assay (total dry weight). Furthermore, STI based on multiple regression analysis gives an accurate definition of salttolerant individuals. Under salt stress, tolerant accessions had high STI and produced higher proline, $\mathrm{K}+$ and $\mathrm{Ca} 2+$, and lower $\mathrm{Na}+$ content than sensitive accessions. Cluster analysis based on related traits to STI, indicated high similarity in each group. These outcomes can be utilized to evaluate the salt tolerance threshold in the species and may have a great advantage over conventional methods. Probably, our upshots can be applied in the next breeding programs to develop salt-tolerant varieties.
\end{abstract}

Keyword: Andrographis paniculata; Salinity stress; Salt tolerance index; Proline; Ion content; Multiple regression model 